01;05

\title{
Средние характеристики ансамбля кластеров кобальта в матрице меди
}

\author{
(ㄱ М.Н. Лубов, Д.В. Куликов, Ю.В. Трушин
}

Санкт-Петербургский национальный исследовательский академический университет РАН, Санкт-Петербург, Россия

E-mail: lubov@spbau.ru

\section{Поступило в Редакцию 3 июля 2018 г.}

Исследован процесс изменения среднего прогиба поверхности меди над подповерхностными кластерами кобальта и доли поверхности, занимаемой ансамблем кластеров. Рассчитаны их зависимости от времени, хорошо согласующиеся с экспериментом.

DOI: $10.21883 /$ PJTF.2018.24.47024.17448

Впервые ансамбль подповерхностных кластеров кобальта в матрице меди при прямом эпитаксиальном осаждении кобальтовых атомов экспериментально получен в работе [1], где были определены структурные характеристики кластеров, в том числе латеральные и вертикальные размеры и концентрация. В работе [2] исследован процесс зарождения и эволюции ансамбля таких кластеров кобальта и рассчитана функция распределения кластеров по размерам. При сравнении экспериментальных данных [1] и функции распределения кластеров кобальта по размерам [2] были оценены неизвестные ранее энергетические параметры в системе кобальт-медь.

Целью настоящей работы являются теоретические оценки экспериментально измеряемых средних величин характеристик ансамблей кластеров кобальта в матрице меди (среднего прогиба поверхности $G(t)$ и ее доли $\Phi(t)$, занимаемой ансамблем кластеров), проведенные на основе разработанной в [2] кинетической модели формирования ансамбля подповерхностных кластеров в системе кобальт-медь и модели трехмерного роста отдельных кластеров кобальта в матрице меди [3].

В эксперименте [1] средняя высота кластеров кобальта $\langle H(t)\rangle$ измеряется по величине среднего прогиба поверхности $G(t)$ над ансамблем кластеров. Как установлено в [1], увеличение средней высоты $\langle H(t)\rangle$ 
кластеров по ансамблю на один монослой приводит к изменению прогиба поверхности над ним на величину $\Delta G \approx 9 \cdot 10^{-12} \mathrm{~m}[1]$. Тогда величина среднего прогиба поверхности $G(t)$ может быть выражена через среднюю высоту кластеров в ансамбле с функцией распределения $f(R, H, t)$ по размерам (радиусам $R(t)$ и высотам $H(t))$ как

$$
G(t)=\frac{(\Delta G / a) \iint H(t) f(R, H, t) d R d H}{\iint f(R, H, t) d R d H}=(\Delta G / a)\langle H(t)\rangle .
$$

Здесь $\langle H(t)\rangle \equiv \iint H(t) f(R, H, t) d R d H / \iint f(R, H, t) d R d H-$ средняя высота кластеров по ансамблю, $a$ - межатомное расстояние в кобальте. Отметим, что кластеры кобальта, полученные в [1], имеют форму, близкую к цилиндрической, поэтому для характеризации латеральных размеров кластера можно использовать величину радиуса $R$ цилиндра соответствующего размера. Доля поверхности, занятая ансамблем кластеров кобальта, определяется как

$\Phi(t) \equiv \frac{\pi \iint R^{2}(t) f(R, H, t) d R d H}{\iint f(R, H, t) d R d H} \iint f(R, H, t) d R d H=\pi\left\langle R^{2}(t)\right\rangle N_{c l}(t)$,

где $\pi\left\langle R^{2}(t)\right\rangle=\pi \iint R^{2}(t) f(R, H, t) d R d H / \iint f(R, H, t) d R d H-$ средняя площадь кластера по ансамблю, $N_{c l}(t)=\iint f(R, H, t) d R d H-$ двумерная концентрация (плотность) подповерхностных кластеров.

Таким образом, величины $\Phi(t)$ и $G(t)$, измеряемые в эксперименте, можно рассчитать теоретически, используя средние площадь $\pi\left\langle R^{2}(t)\right\rangle$ и высоту $\langle H(t)\rangle$ кластеров по ансамблю, а также их плотность $N_{c l}(t)$.

Изменение плотности подповерхностных кластеров $N_{c l}(t)$ и их размеров $(R(t)$ и $H(t))$ со временем можно оценить исходя из кинетики взаимодействий атомов кобальта при росте немонослойного кластеpa $[2,3]$.

Изменение плотности подповерхностных атомов кобальта $N_{\text {со }}(t)$ происходит при переходе в подповерхностные слои атомов кобальта

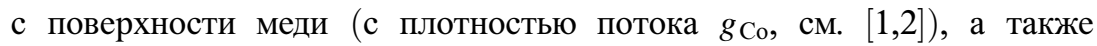
при присоединении подповерхностных атомов кобальта к растущим кластерам кобальта (с плотностью потока $\left.g_{\mathrm{Co}}^{c l}(t)\right)$ :

$$
d N_{\mathrm{Co}}(t) / d t=g_{\mathrm{Co}}-g_{\mathrm{Co}}^{c l}(t),
$$

где $g_{\mathrm{Co}}^{c l}(t)=\beta_{\mathrm{Co}}(t) D_{\mathrm{Co}} N_{\mathrm{Co}}(t) N_{c l}(t)$ - частота актов присоединения атомов кобальта к подповерхностным кластерам кобальта, $D_{\text {Со }}$ 
коэффициент диффузии атомов кобальта в подповерхностном слое, $\beta_{\mathrm{Co}}(t)$ - параметр, учитывающий эффективность захвата подповерхностных атомов кобальта кластером среднего радиуса $\langle R(t)\rangle$ и зависящий как от периметра кластера $2 \pi\langle R(t)\rangle$ (определяет количество мест на краю кластера, куда может присоединиться атом кобальта), так и от площади поверхности кластера $\pi\left\langle R^{2}(t)\right\rangle$ (определяет площадь зоны „питания“ кластера, т. е. области, откуда атомы кобальта диффундируют к кластеру) [4-8].

Изменение доли поверхности $\Phi(t)$, занятой подповерхностными кластерами кобальта среднего радиуса $\langle R(t)\rangle$, можно оценить исходя из оценки скорости присоединения подповерхностных атомов кобальта к краю первого (верхнего) слоя кластера с вероятностью $P_{1}$ в виде

$$
d \Phi(t) / d t=a^{2} P_{1} g_{\mathrm{Co}}^{c l}(t) .
$$

При этом нужно учесть, что для атомов кобальта должно выполняться условие для вероятностей его присоединения к верхнему слою кластера $P_{1}$ и к остальным слоям кластера $P$ в виде $P_{1}+P=1$.

Высота подповерхностного кластера $H(t)$ увеличивается при присоединении одного атома кобальта к слоям ниже верхнего на величину

$$
\Delta H(t)=a^{3} / \pi\left\langle R^{2}(t)\right\rangle,
$$

при этом радиус кластера остается неизменным. Таким образом, среднюю высоту подповерхностных кластеров можно выразить как $\langle H(t)\rangle=a+n(t) \Delta H(t)$, где $n(t)$ - число атомов кобальта, находящихся не в верхнем слое подповерхностного кластера. Их изменение со временем можно оценить как

$$
d n(t) / d t=\frac{P}{N_{c l}(t)} g_{\mathrm{Co}}^{c l}(t) .
$$

Тогда для величины среднего прогиба $G(t)$ поверхности меди над кластерами на основе выражений (1) и (5) можно записать

$$
G(t)=\Delta G\left(1+\frac{n(t) a^{2}}{\pi\left\langle R^{2}(t)\right\rangle}\right) .
$$

Подставив выражение (2) для доли поверхности $\Phi(t)$, занятой подповерхностными кластерами, в (4) и учитывая временну́ю зависимость

Письма в ЖТФ, 2018, том 44, вып. 24 
плотности кластеров $\mathrm{N}_{c l}(\mathrm{t})$ (см. [2]), получим уравнение для средней площади подповерхностного кластера в виде

$$
\frac{d\left[\pi\left\langle R^{2}(t)\right\rangle N_{c l}(t)\right]}{d t}=a^{2} P_{1} g_{\mathrm{Co}}^{c l}(t)
$$

Выражения (4) и (7) позволяют найти зависимости от времени соответственно доли поверхности $\Phi(t)$, занятой подповерхностными кластерами кобальта среднего радиуса $\langle R(t)\rangle$, и среднего прогиба $G(t)$ над кластерами, решая замкнутую систему балансных уравнений $(3)-(8)$. Вероятности $P_{1}$ и $P$ определяются частотами $v_{1}$ и $v$ присоединения атомов кобальта к кластеру кобальта

$$
P_{1}=v_{1}\left(v_{1}+v\right)^{-1}, \quad P=v\left(v_{1}+v\right)^{-1} .
$$

В соответствии с результатами работы [3] частоты $v_{1}$ и $v$ зависят от выигрыша в энергии за счет появления связей атомов кобальта друг с другом при присоединении атома кобальта к кластеру, а также от проигрыша в упругой энергии за счет увеличения радиуса или высоты кластера. Увеличение радиуса кластера приводит к уменьшению величины $v_{1}$ и возрастанию величины $v$; увеличение же высоты кластера приводит к возрастанию $v_{1}$ и уменьшению $v$ (см. выражения (10) и (12) в [3]). Кроме того, присоединение атомов кобальта к нижним слоям кластера затрудняется вследствие увеличения барьера активации миграции с глубиной проникновения кобальта в медь (см. [3]).

На рис. 1 и 2 соответственно представлено сравнение с экспериментальными данными работы [1] результатов расчетов величин $\Phi(t)$ и $G(t)$ в зависимости от номинального количества осажденного кобальта $F=g_{\text {со }} t=3.6 \cdot 10^{-3} \mathrm{ML} / \mathrm{s} \cdot t \quad$ (см. [2]). Расчеты проводились при следующих значениях параметров: $T=650 \mathrm{~K}$ [1], величина

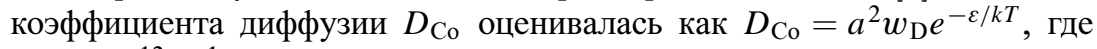
$w_{\mathrm{D}}=10^{12} \mathrm{~s}^{-1}$ - частота порядка дебаевской, $\varepsilon=1.29 \mathrm{eV}-$ энергия миграции атомов кобальта в подповерхностной области меди [2]. Из рис. 1 и 2 следует, что результаты моделирования удовлетворительно согласуются с экспериментом.

Таким образом, разработанная в [2,3] кинетическая модель роста подповерхностных кластеров кобальта в меди адаптирована для моделирования эволюции средних величин радиуса и высоты по ансамблю подповерхностных кластеров. С помощью этой модели рассчитаны

Письма в ЖТФ, 2018, том 44, вып. 24 


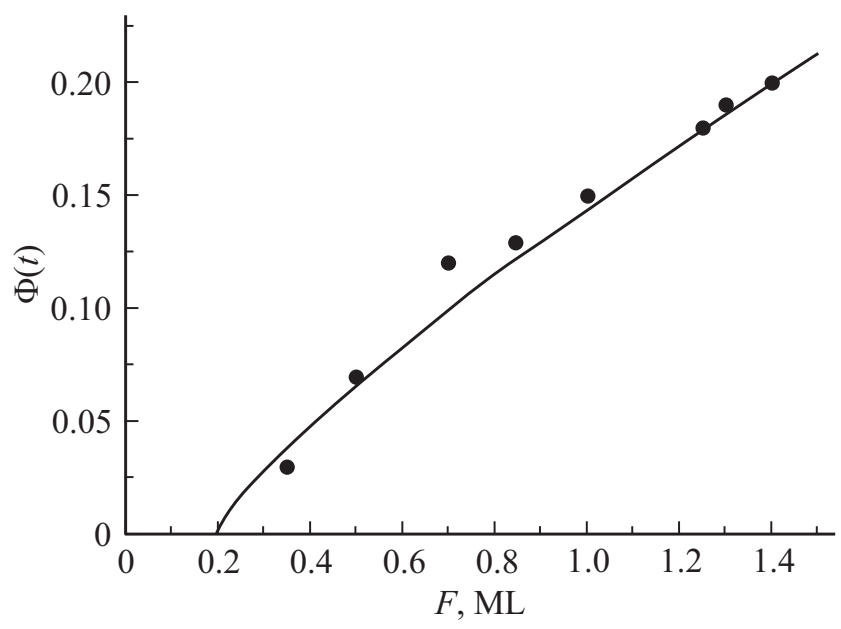

Рис. 1. Зависимость доли поверхности $\Phi(t)$, занятой кластерами кобальта, от номинального количества осажденного кобальта $F$. Точки - экспериментальные данные [1], линия - расчет.

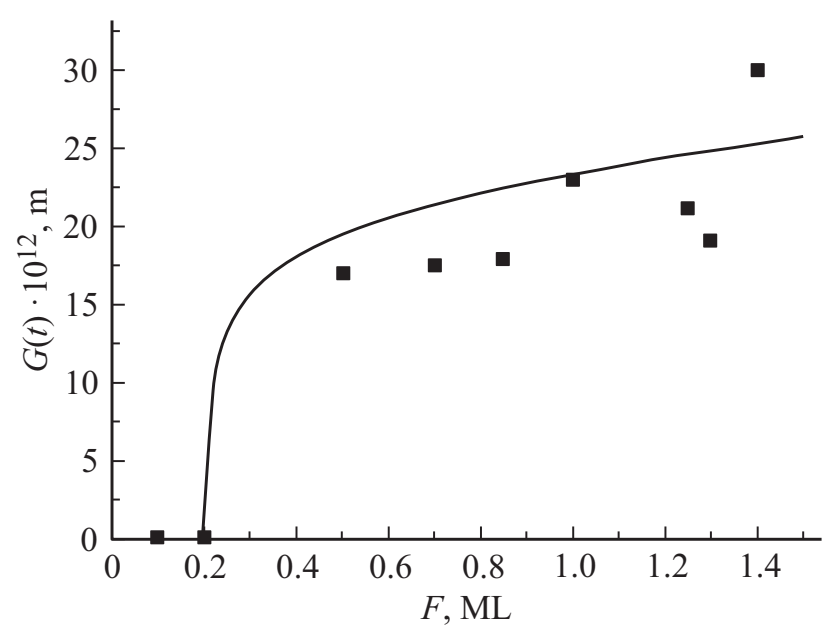

Рис. 2. Зависимость среднего прогиба $G(t)$ поверхности меди над кластерами кобальта от номинального количества осажденного кобальта $F$. Точки экспериментальные данные [1], линия - расчет.

Письма в ЖТФ, 2018, том 44, вып. 24 
зависимости от времени для доли поверхности $\Phi(t)$, занятой подповерхностными кластерами, и для среднего прогиба $G(t)$ поверхности меди над подповерхностными кластерами, которые согласуются с экспериментальными данными [1]. Это позволяет заключить, что модель удовлетворительно описывает кинетику заполнения кластерами кобальта подповерхностной области меди, а также дает возможность выбирать оптимальные технологические режимы выращивания ансамблей магнитных кластеров в металлической матрице.

Авторы выражают благодарность О. Курносикову за предоставление экспериментальных данных и полезные обсуждения.

Часть работы, выполненная М.Н. Лубовым, осуществлена в рамках государственного задания Министерства образования и науки РФ № 16.9789.2017/БЧ.

\section{Список литературы}

[1] Siahaan T., Kurnosikov O., Swagten H.J.M., Koopmanset B. // Phys. Rev. B. 2014. V. 90. P. 165419.

[2] Lubov M., Kulikov D.V., Trushin Yu. // Phys. Status. Solidi B. 2017. V. 254. P. 1700250.

[3] Лубов М.Н., Куликов Д.В., Курносиков О., Трушин Ю.В. // Изв. РАН. Сер. физ. 2014. Т. 78. В. 6. С. $682-685$.

[4] Smoluchowki M. // Phys. Z. 1916. V. 17. P. 557-585.

[5] Bartelt M.C., Stoldt C.R., Jenks C.J., Thiel P.A., Evans J.W. // Phys. Rev. B. 1999. V. 59. P. 3125-3134.

[6] Amar J.G., Popescu M.N., Family F. // Phys. Rev. Lett. 2001. V. 86. P. 3092-3095.

[7] Trushin Yu.V., Kulikov D.V., Safonov K.L., Gerlach J.W., Höche Th., Rauschenbach B. // J. Appl. Phys. 2008. V. 103. P. 114904.

[8] Pezoldt J., Kulikov D.V., Kharlamov V.S., Lubov M.N., Trushin Yu.V. // J. Comput. Theor. Nanosci. 2012. V. 9. P. 1941-1966. 